\title{
Gas phase ion chemistry: what do we know about reactions and ion formation?
}

\author{
Helko Borsdorf ${ }^{1} \cdot$ Robert G. Ewing ${ }^{2}$
}

Received: 12 April 2015 / Accepted: 24 April 2015 / Published online: 10 May 2015

(C) Springer-Verlag Berlin Heidelberg 2015

It is certainly the case that numerous sensor techniques operate based on measuring principles which are far less complex than those associated with ion mobility spectrometry (IMS). However, the additional complexity of IMS provides a higher level of quantitative and qualitative information. The ion mobility measurements provide characteristic spectra for investigated samples and do not merely provide a sum signal. The results are available within seconds and contain a high level of information density with excellent detection limits for numerous chemical substances.

The formation of ions from neutral sample molecules is the first and possibly the most significant event during ion mobility measurements. The ionization of a sample occurs in air or nitrogen at ambient pressure. This atmospheric pressure chemical ionization (APCI) provides both the sensitivity and selectivity achieved by IMS. The most common APCI source used in IMS analyzers today is a radioactive ionization source, typically 100 or $370 \mathrm{MBq}$ of ${ }^{63} \mathrm{Nickel}\left({ }^{63} \mathrm{Ni}\right)$ or Tritium $\left({ }^{3} \mathrm{H}\right)$. Unfortunately, these ion sources with radioactive isotopes require special use permits and licensing procedures. Moreover, the freedom to transport such analyzers is limited by certain legal constraints and semi-annual leak tests represent an additional cost and burden of ownership. For these reasons, nonradioactive ion sources have been explored and developed as alternatives to traditional radioactive sources. These developments led to the discovery of a variety of ion sources which

Helko Borsdorf

helko.borsdorf@ufz.de

1 Department Monitoring and Exploration Technologies, UFZ Helmholtz Centre for Environmental Research, Permoserstraße 15, D-04318 Leipzig, Germany

2 Pacific Northwest National Laboratory, Richland, WA 99352, USA can be used for gas sensing with IMS. More than 10 different ionization principles are described in recent review articles and monographs for the measurement of gaseous compounds [H. Borsdorf, T. Mayer, M. Zarejousheghani, G.A. Eiceman, Appl. Spectrosc. Rev. 46 (2011) 472-521; G.A. Eiceman, Z. Karpas, H.H. Hill, Ion Mobility Spectrometry, Third Edition, Taylor \& Francis Group, 2013]. Each of these ion sources provides different ionization pathways and ionization reactions. Additionally, the gas phase reactions during and after ionization are considerably affected by experimental parameters (e.g., humidity, temperature, pressure). In particular, differences in temperature and humidity can result in the formation of different reactant ions and product ions. Furthermore, the sample matrix and accompanying substances can affect the gas phase reactions in IMS. All of these parameters not only influence the nature of ions formed during gas phase ion chemistry, but also affect their relative abundance depending on the experimental conditions and sample matrix. As can be seen, gas phase ion chemistry is quite complex and the prediction of possible reaction pathways requires the consideration of numerous parameters. As we move away from radioactive ionization sources and develop new ionization techniques, the study of the ionization chemistry is critical both in maintaining the ability to detect analytes in existing IMS applications as well as the development of methods to detect emerging threats.

While IMS was solely used as a gas-phase analyzer in preceding decades, further developments, especially in electrospray ionization and its derivatives, now permit it to be routinely used for the analysis of liquid samples. These ion sources use high electric fields on a solution as a means to ionize polar non-volatile substances using different mechanisms than those in APCI techniques.

However, ion chemistry does not stop in front of the shutter grid. Reactions can also occur as ions travel through the 
analyzer. In traditional time-of-flight IMS, these reactions can result from the interaction between ions and drift gas molecules, as well as among ions themselves. Such gas phase reactions are more significant in field-dependent methods. The high electric fields in differential mobility spectrometers (DMS) or high-field asymmetric waveform ion mobility spectrometer (FAIMS) methods induce ion heating by energetic collisions with buffer gas molecules, which may encourage isomerization or dissociation. The formation and dissociation of ion clusters occurs repeatedly at high frequencies in fielddependent methods. Both deformation and variation of crosssections in high electric fields have also been extensively investigated.

The most recent reviews describe 8 different types of ion mobility analyzers [R. Cumeras, F. Figueras, C.E. Davis, J.I. Baumbach, I. Gracia, Analyst 140 (2015) 1376-1390]. All of these analyzers are characterized by special flow regimes, different vacuum states, different field strengths and field gradients. Depending on experimental conditions, additional ionmolecule reactions can occur within the analyzer having varying degrees of effects on the results.

What do we know about gas phase reactions in IMS? In our opinion: not as much as we should. Gas phase ion chemistry is easily described when a substance has "ideal" properties of low ionization energies (for photoionization) or high proton affinities (for chemical ionization). Complications with predicting the response in a mobility spectrometer arise with variations in the experimental parameters cited above. It is difficult in practice to realize the whole complexity of gas phase ion chemistry within an ion mobility spectrometer. Why is it that we can detect substances with ${ }^{63} \mathrm{Ni}$ ionization despite the fact that proton transfer reactions are unlikely according to their physicochemical properties?

Mass spectrometry has been used to assist in the understanding of ion formation in an ion mobility spectrometer. However, does the coupling of IMS with mass spectrometry (IMMS) answer all of our questions? The ion mobility spectrometer used in most of the commercially-available IMMS instruments works at a reduced pressure and the processes are not comparable with those of an IMS-based gas sensing device operating at ambient pressure. For an ion mobility spectrometer operating at atmospheric pressure, the interface to the mass spectrometer is accomplished with a pinhole or a capillary. To acquire enough signal intensity with the smaller orifice, the measurements usually require a longer shutter grid opening time and a higher concentration range of compounds than is normally used as for IMS alone. A lack of knowledge exists regarding the stability of ions or ion clusters during transition from the ambient pressure of IMS to the high vacuum state of MS. Further reactions in long capillary interfaces may also occur. Although IMMS is a helpful tool for the clarification of gas phase ion chemistry, it does not solve all of the previously-mentioned issues raised.

Atmospheric pressure ion chemistry is a complex process and there are many unanswered questions and unknown aspects concerning its use. We cannot resolve all of the previously-mentioned problems. However, the main question is: What ions are generated in our ion mobility spectrum and why? This curiosity drives us on to explore the unknown.

Finding answers to this question and obtaining the required information is essential for the validation of IMS-based methods and of possible matrix effects.

This special issue provides a mix of original articles which highlight various aspects of ion-molecule interactions. The ion chemistry for different ion sources is described; the influence of operational parameters on the ionization processes is also discussed, as is the ion chemistry of special compounds in both the gaseous and liquid phase. We have endeavored to provide a balanced and comprehensive survey. However, not all of the aforementioned topics can be tackled in great detail.

We thank all of the authors for their interesting contributions. We are also very thankful to the reviewers for their valuable support and for providing critical and constructive reviews.

Enjoy reading!

Sincerely,

Helko Borsdorf and Robert Ewing 